\title{
The Effect of Applied Vertical Magnetic Field on Anodizing Behavior of Aluminum to Produce Porous Anodic Aluminum Oxide
}

\author{
Sigit Wahyu Prabowo, ${ }^{*}$ Shinjiro Fukuda, Kenta Uchida, ${ }^{1}$ \\ Takeshi Hashishin, ${ }^{2}$ and Toshiyuki Kozuka ${ }^{2}$ \\ Graduate School of Science and Technology, Kumamoto University, \\ 2-39-1 Kurokami, Chuo-ku, Kumamoto 860-8555, Japan \\ ${ }^{1}$ Department of Material Science and Engineering, Faculty of Engineering, Kumamoto University, \\ 2-39-1 Kurokami, Chuo-ku, Kumamoto 860-8555, Japan \\ ${ }^{2}$ Division of Material Science, Functional Material Engineering, Kumamoto University, \\ 2-39-1 Kurokami, Chuo-ku, Kumamoto 860-8555, Japan
}

(Received June 28, 2016; accepted August 3, 2016)

Keywords: anodic oxidation, anodic aluminum oxide, magnetic field, template, sensor

Porous anodic aluminum oxide (AAO) film has potential to be a candidate material in nanofabrication, membranes, and humidity sensors. Anodic oxidation is typically preferred to fabricate AAO because it is an inexpensive and controllable process. In this study, a different approach to fabricating AAO by a single step anodizing in oxalic acid assisted by externally applied vertical magnetic field, is adopted. The anodizing experiments were conducted in $0.3 \mathrm{M}$ oxalic acid aqueous solution as electrolyte. The vertical magnetic field was generated by a superconducting magnet with the range of 1 to $5 \mathrm{~T}$. The results of the experiment showed that the magnetic field can promote the anodic dissolution of $\mathrm{Al}^{3+}$ ions, and hence increase the AAO thickness by twice as much as without one. However, the pore diameter was decreased by the increased magnitude of the magnetic field. Interestingly, the AAO formed by anodizing with the vertical magnetic field showed a vertically aligned cylindrical structure of the pores. On the other hand, AAO formed by anodizing without the magnetic field showed random growth of pore structure. The density of AAO pores was also increased by the increase in magnitude of the magnetic field. The deep and straight structure with high density and porosity of AAO is advantageous for further application as template and sensor materials.

\section{Introduction}

The protective passive oxide film of aluminum which exhibits remarkable properties such as dielectric properties, thermal stability, adhesive, wear, and corrosion resistance can be produced by anodic oxidation treatment. ${ }^{(1)}$ Anodic oxidation is also used to produce similar oxide film for other valve metals like titanium and silicon. ${ }^{(2,3)}$ In general, there are two types of oxide films, namely barrier-type oxide film and porous-type oxide film, depending on the electrolyte and anodizing conditions. Compared to barrier-type film, the porous alumina film called anodic aluminum oxide (AAO) has attracted great interest because it contains nano-sized pores throughout the whole area ${ }^{*}$ Corresponding author: e-mail: sigitwahyuprabowo@gmail.com 
of the aluminum substrate. This porous AAO film has potential to be used as a template for the fabrication of nanostructured materials, such as nanotubes, nanowire, and nanodots. ${ }^{(4,5)}$ Since the anodic oxidation process is relatively inexpensive, much effort has been made by researchers to replace the expensive photolithography method with AAO film. ${ }^{(6)}$ Owing to its high mechanical and chemical stability, AAO can also be utilized as a starting material in chemical sensors and humidity sensors by exploiting its porous structure..$^{(7-9)}$

The aluminum oxide film can also be fabricated by the sol-gel method, chemical vapor deposition, or thermal oxidation. ${ }^{(10,11)}$ However, anodic oxidation is preferable because it is considerably less expensive, and a more controllable process. In respect to its structure, the cylindrical pore structures are the important structures for further application, due to the highly ordered array of vertical stands on the surface of aluminum substrate. ${ }^{(12)}$ There are a number of reports about producing such a structure by direct imprinting of the substrate using a stamp mold, or direct tipping by a scanning probe electron microscope to make a concave pattern on the surface prior to anodizing. ${ }^{(13)}$ However, the cost of manufacturing using this method is considerably high, time consuming, and produces only a few square millimeters of AAO (limited area). The other method is the two-step anodization method first introduced by Masuda and Fukuda.(14) Although this method is considerably less expensive, it has major disadvantages such as the need for strict temperature control $\left(1-5{ }^{\circ} \mathrm{C}\right)$, low porosity $(3.3-10 \%)$, and slow porous growth. ${ }^{(13,15)}$

In the current method of material processing, the application of an electric or magnetic field into the process results in the electromagnetic force, reduces surface defects, eliminates the inclusion in steel, and induces crystal structure alignment such as finer crystal.(16) In electrochemical processing, the advantage of the magnetic field is improving mass transport, accelerating oxidation reaction at electrode, and assisting the rate of passivation on the surface. ${ }^{(17)}$ The main effect of the applied magnetic field on the electrochemical reaction is the additional magnetic convection in the electrolyte, called the magnetohydrodynamic (MHD) effect. This convection results from the Lorentz Force generated by the interaction of the magnetic field perpendicular to the current line. In electrodeposition of copper, it was reported that the MHD effect increases the current density and promotes mass transfer to the electrode. ${ }^{(18)}$ The additional effect, called micro-MHD, was also observed in the electrodeposition of copper, which resulted in numerous microscopic vortices in the vicinity of the precipitation interface on the surface of the cathode and suppressed crystal growth of electrodeposited $\mathrm{Cu}$ on the cathode. ${ }^{(19,20)}$ Meanwhile, there remain few reports of the application of a magnetic field to anodic oxidation. ${ }^{(21)}$ In this study, the effect of magnetic field imposition on the surface morphology of anodized oxide film was investigated for various magnetic fields. The main purpose is controlling the AAO microstructures, i.e., pore size and pitch, by one-step anodization assisted by a vertical magnetic field.

\section{Materials and Methods}

An aluminum sheet, which had a square size of $4 \mathrm{~cm}^{2}, 99.99 \%$ purity, and a thickness of 1.2 $\mathrm{mm}$, was used as a starting substrate. The substrates were then sonicated in acetone for $3 \mathrm{~min}$ and dried to remove contamination on the surface. The cleaned substrates were then immersed in $3 \mathrm{M}$ $\mathrm{NaOH}$ aqueous solution for $3 \mathrm{~min}$ and dipped in $3 \mathrm{M} \mathrm{HNO}_{3}$ aqueous solution for $10 \mathrm{~s}$ to remove the natural oxide layer on the aluminum surface. Then, the treated substrates were electro-polished at $20 \mathrm{~V}$, at $5{ }^{\circ} \mathrm{C}$, for $3 \mathrm{~min}$ in a mixture of $60 \% \mathrm{HClO}_{4}$ and $\mathrm{C}_{2} \mathrm{H}_{5} \mathrm{OH}$ prepared at the volume ratio of $1: 4$ to obtain a mirror-like surface. 
In the anodizing experiment, the prepared $\mathrm{Al}$ sheet was used as the anode, and a platinum $(\mathrm{Pt})$ plate with a square area of $1 \mathrm{~cm}^{2}$ was used as the cathode. The anodizing cell was composed from polytetrafluoroethylene (PTFE). The distance between the anode and the cathode was set to approximately $1 \mathrm{~cm}$, and an O-ring was used for limiting the exposed anodized area [Fig. 1(b)]. The vertical magnetic field was imposed during anodization. The anodizing cell was set in the magnet bore, a superconducting magnet HF5-100VT-50H-1 manufactured by Sumitomo Heavy Inc. Figure 1(a) shows the cross sectional view of the total system for the anodizing equipment located in the center of the magnetic field inducer. The superconducting magnet comprises four magnetic coils which generate a uniform magnetic field in the center part of the magnet bore. The magnetic field direction is downward and is denoted as $B$ in Fig. 1(a). A wooden stand was used to set the anodizing cell in the center where the magnetic field was uniformly maintained. The aluminum anodizing was conducted in $0.3 \mathrm{M}$ oxalic acid solution for $4 \mathrm{~h}$ under the potential of $50 \mathrm{~V}$. The anodizing was performed at room temperature and in a single step. The strength of the imposed magnetic field ranged from 1 to $5 \mathrm{~T}$.

The nanostructure observation of the obtained porous aluminum oxide was performed by using a field emission scanning electron microscope (FE-SEM, JEOL, JSM-7600F). The crystal phase characterization was performed by thin film X-ray diffraction (XRD Rigaku, RINT2500) with $\mathrm{CuK} \alpha$ radiation at a scan rate of $1^{\circ} \mathrm{min}^{-1}$. The structural properties of porous AAO were measured by Gatan Digital Micrograph software.

\section{Results and Discussion}

Figure 2 shows the high resolution FE-SEM images of porous AAO anodized in oxalic acid at $50 \mathrm{~V}$ for $4 \mathrm{~h}$ at various magnetic flux densities. The porous structure of AAO formed by imposition of the vertical magnetic field was apparently uniform without increased temperature and no oxide burning. The diameter of pores tended to decrease with the increase in the magnitude of the vertical magnetic field. A significant decrease of pore size in the average diameter was observed for increasing the magnetic field up to $1 \mathrm{~T}$, from 93 to $72 \mathrm{~nm}$, and a slight decrease of pore diameter was observed by a higher magnetic field up to $5 \mathrm{~T}$, as shown in Fig. 3.

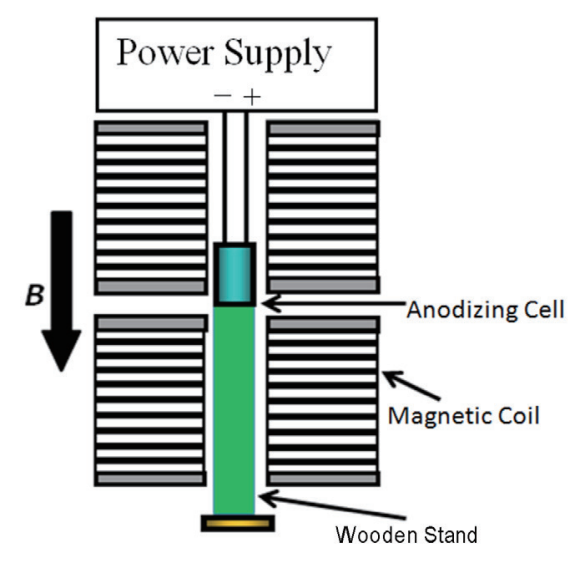

(a)

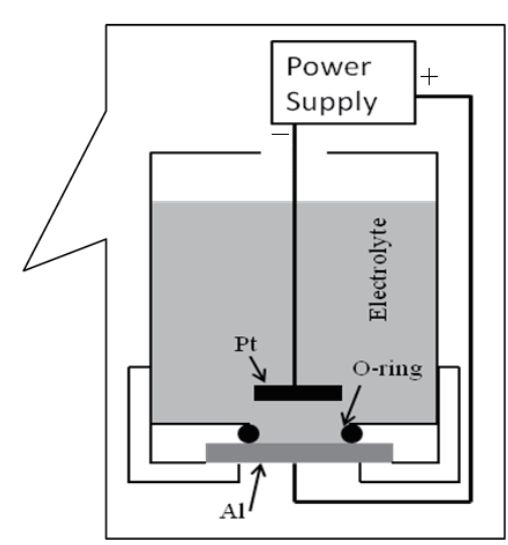

(b)

Fig. 1. (Color online) Schematic illustration for the cross sectional view of the (a) anodizing experimental setup in a magnet bore and (b) anodizing cell. 


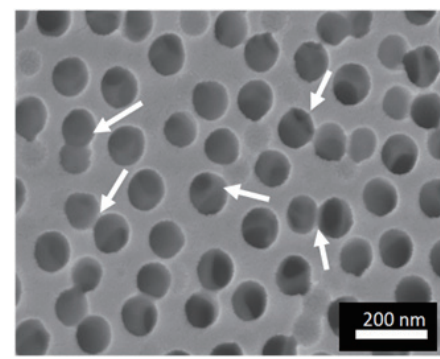

(a)

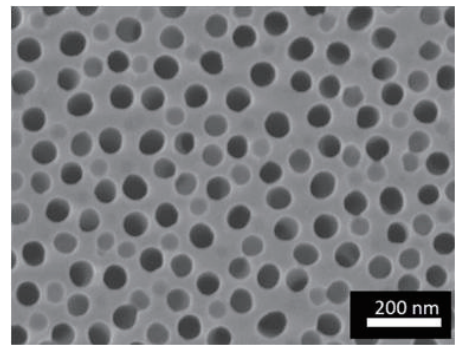

(c)

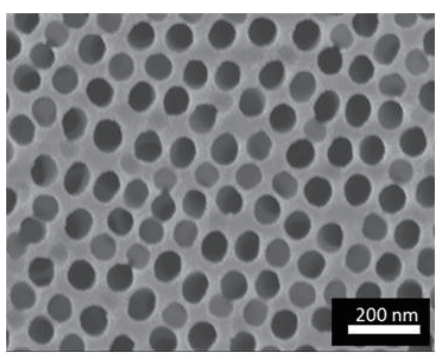

(b)

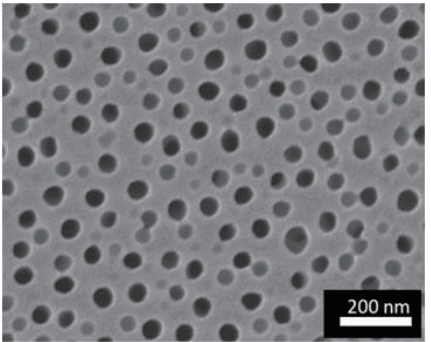

(d)

Fig. 2. FE-SEM images of the AAO surface with the applied potential of $50 \mathrm{~V}$ with (a) no magnetic field and with the magnetic field of (b) 1 , (c) 3 , and (d) $5 \mathrm{~T}$.

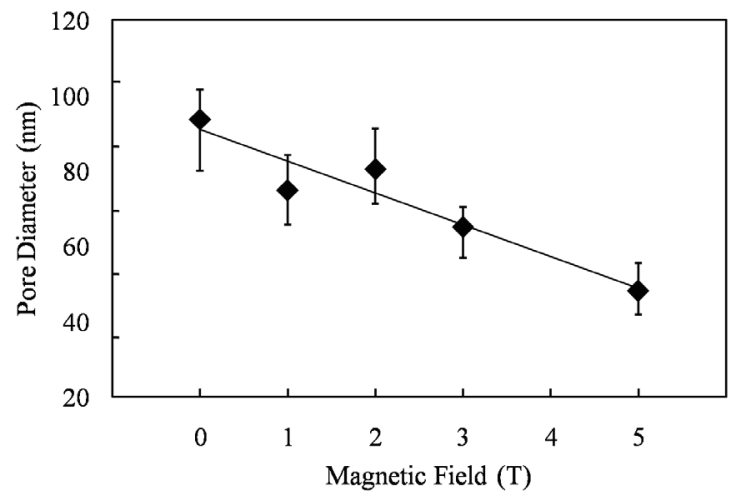

Fig. 3. Effect of the magnetic field on the average pore diameter of AAO formed in $0.3 \mathrm{M}$ oxalic acid at $50 \mathrm{~V}$ for $4 \mathrm{~h}$.

In aluminum anodizing, single-step anodizing generally results in a random pattern of pores. Similar behavior is also shown in Fig. 2(a) in which the porous AAO was obtained by anodizing in constant potential without an imposed magnetic field. The distribution of AAO pores is not formed orderly, which can be seen from the wide error-bar of inter-pore distance in Fig. 4. There were pores which conjugate each other, and so the pore spacing was largely varied. Moreover, the side-wall of pores can be seen under the top of the pores, meaning that these pores were grown disorderly [white arrow in Fig. 2(a)]. The morphology of AAO pores obtained by imposition of vertical magnetic field exhibits fairly ordered distribution and more circularity, as in shown Figs. 2(b)-2(d). This ordered distribution of pores can be seen as a narrow error bar of the interpore distance (Fig. 4). The inter-pore distance was decreased by increasing the magnitudes of the magnetic field. The constancy of the inter-pore distance showed that pore centers do not shift, even with increased magnetic field. 


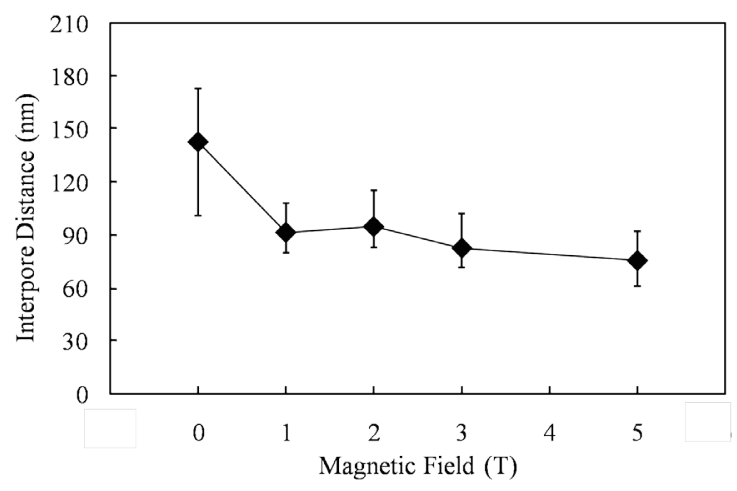

Fig. 4. Effect of the magnetic field on the average interpore distance of AAO formed in $0.3 \mathrm{M}$ oxalic acid at $50 \mathrm{~V}$ for $4 \mathrm{~h}$.

The imposed magnetic field produces more densely AAO nanopores than without it. The pore density of Fig. 5 was significantly increased by imposing a magnetic field. Pore density was calculated by the total number of pores divided by the total area from the FE-SEM image. The pores tended to be smaller with increasing magnetic field, as seen in Fig. 2. Moreover, the interpore distance decreased and was widely distributed, as seen in Fig. 4. Thus, it was expected that more pores are formed on the surface. Another important parameter of AAO is pore fraction or porosity. This parameter was evaluated as pore fraction, in Fig. 6, i.e., the total pore area divided by the total surface area from the FE-SEM image. The average pore fraction, shown by solid diamond shapes in Fig. 6, fluctuated with increases in magnetic field. The pore fraction of AAO indicated the volcano-type tendency, which reached the peak value of ca. $46 \%$ at $1 \mathrm{~T}$ or $2 \mathrm{~T}$, and decreased to $29 \%$ at $5 \mathrm{~T}$. This tendency was related to the constancy of the inter-pore distance and the decrease of pore diameter with increasing magnetic field. For example, in the absence of magnetic field, the inter-pore distance was large, while in the presence of magnetic field $(1 \mathrm{~T})$, the pore distance was smaller (Fig. 4) and denser (Fig. 5). Hence, the pore fraction increased (Fig. 6). By increasing the magnitude of the magnetic field, the pore diameter was decreased, with no significant changes in inter-pore distance. Hence, the pore fraction was decreased. From the above tendencies, it was found that the porosity became higher by anodizing in oxalic acid under a vertical magnetic field of $1 \mathrm{~T}(46.4 \%)$. Obtaining highly dense, compact and porous AAO film is advantageous for further application in nanofabrication and sensors.

Figure 7 shows the cross sectional view of AAO formed by anodizing in $0.3 \mathrm{M}$ oxalic acid for $4 \mathrm{~h}$ at the tip of the surface and at the pore bottom. Here, the cylindrical structure represents the porous AAO. As can be seen from the surface image [Fig. 2(a)], the cross sectional morphologies deviated from straight-growth during anodization without a magnetic field, leading to disordered pores, as shown in Fig. 7(a). From the cross sectional view of the bottom, it seems that the AAO formed by anodizing without magnetic field showed a porous growth in random directions, as shown in Fig. 7(d). These morphologies of the surface and bottom of AAO tend to be normally observed in single step anodizing without magnetic field. The disordered alignment of AAO could be attributed to the localized electric field at the initial stage of pore formation which involved the breakdown of the barrier layer by localized electric field distribution at breakdown sites. The nonuniform electric field leads to random growth of pores because electric field distribution prefers a 


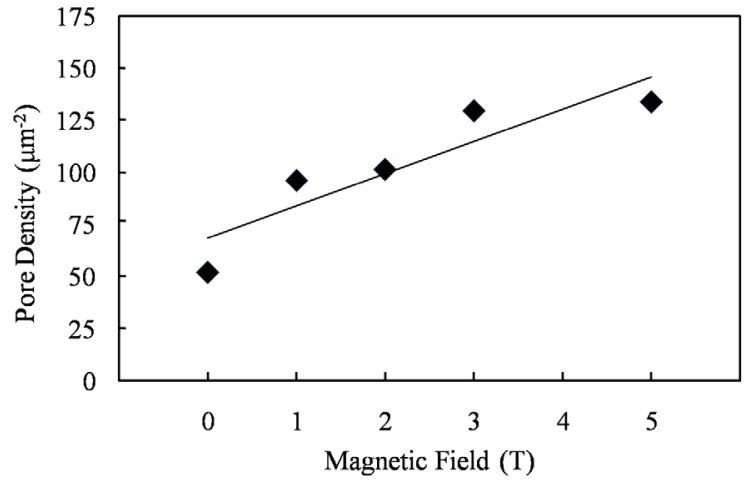

Fig. 5. Effect of the magnetic field on the pore density of AAO formed in $0.3 \mathrm{M}$ oxalic acid at $50 \mathrm{~V}$ for $4 \mathrm{~h}$.

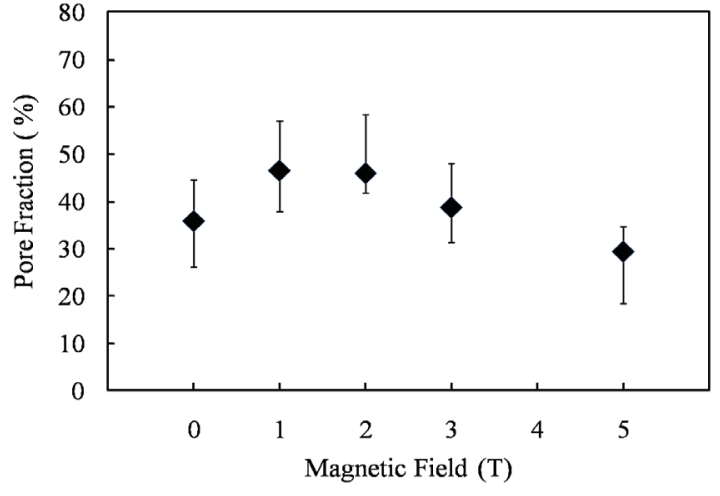

Fig. 6. Average pore fraction of AAO formed in 0.3 $\mathrm{M}$ oxalic acid at $50 \mathrm{~V}$ for $4 \mathrm{~h}$ in various magnitudes of magnetic field.

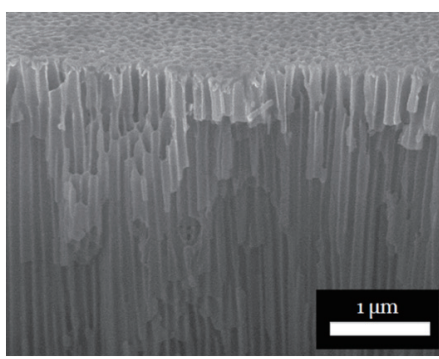

(a)

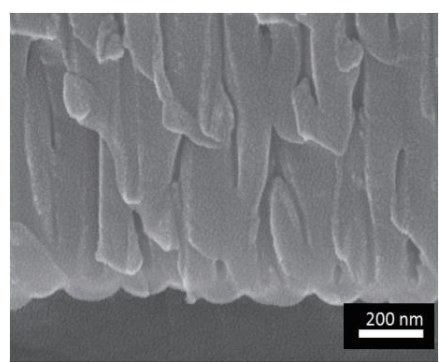

(d)

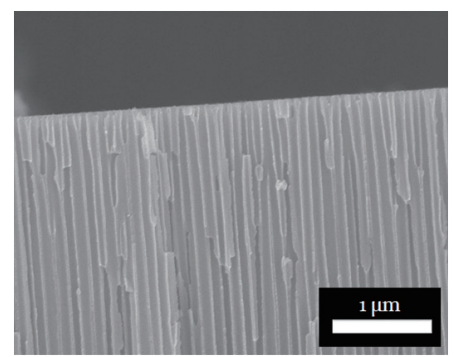

(b)

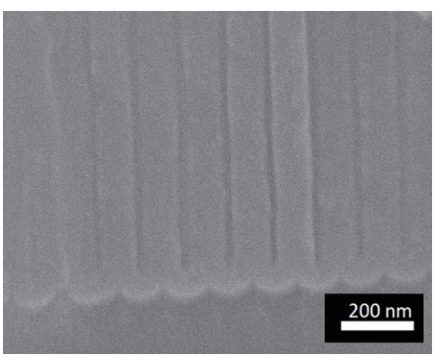

(e)

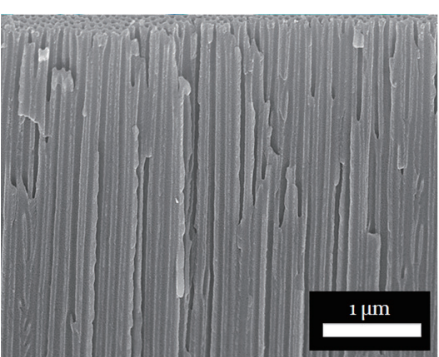

(c)

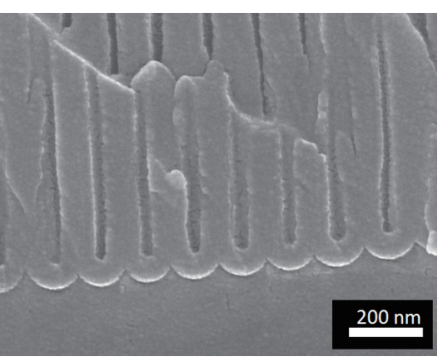

(f)

Fig. 7. FE-SEM images of the cross sectional view at the $(\mathrm{a}-\mathrm{c})$ top and $(\mathrm{d}-\mathrm{f})$ bottom of AAO anodized without magnetic field (a, d) and with the magnetic field of $1 \mathrm{~T}(\mathrm{~b}, \mathrm{e})$ and $5 \mathrm{~T}(\mathrm{c}, \mathrm{f})$.

site with lower resistivity. The pore structure of AAO formed by anodizing with magnetic field was vertically aligned, even in single steps, as shown in Figs. 7(b), 7(c), 7(e), and 7(f). This indicates that the MHD effect resulting from the applied magnetic field occurred and could assist the straightgrowth of AAO with cylindrical porous structure.

The effect of a vertical magnetic field on the thickness of AAO is presented in Fig. 8. In the absence of a magnetic field, the average AAO thickness is $51.35 \mu \mathrm{m}$, and with increasing magnetic field, the average AAO thicknesses are 98.17, 105.0, 103.3, and $93.8 \mu \mathrm{m}$ in 1, 2, 3, and $5 \mathrm{~T}$ respectively. The porous AAO thickness was increased as much as twice that of AAO obtained without a magnetic field, with no significant changes with increasing magnetic field. This 


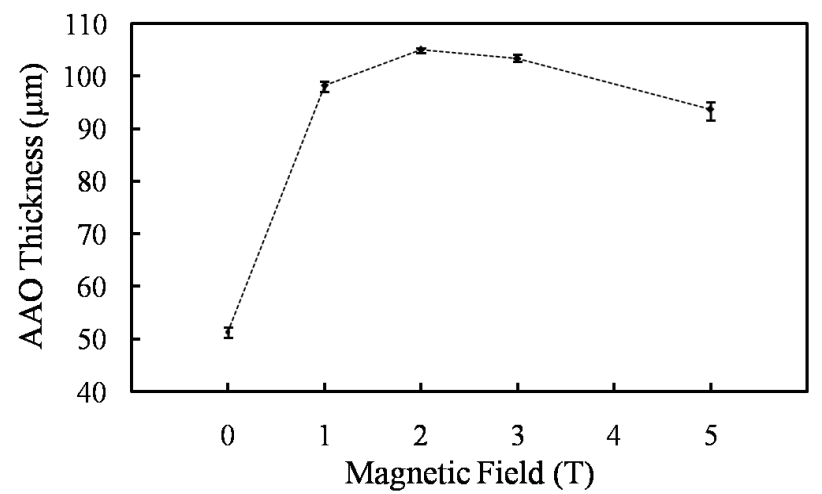

Fig. 8. Effect of the magnetic field on the thickness of AAO formed in $0.3 \mathrm{M}$ oxalic acid at $50 \mathrm{~V}$ for $4 \mathrm{~h}$.

means that the MHD effect promotes the growth of AAO pores. However, the high intensity of the magnetic field did not seem to produce a significant increase in the growth of pores. A small decrease of AAO thickness was observed at magnetic field of $5 \mathrm{~T}$. It seems that a strong magnetic field $(5 \mathrm{~T})$ resulted in strong magnetic convection on the electrolyte and could disturb the effect on porous growth. Hence the growth of pores is slightly decreased. The AAO formed with imposed magnetic field has a large thickness, a high aspect ratio $(>1380)$, and a straight structure. The deep and straight pore structure contributes to the increase in the amount of adsorption of gases due to the larger specific area of AAO. This is essential for the application as a template to produce perfect structures of nanotube or nanowire, and also for sensing properties such as in humidity sensors for $\mathrm{H}_{2} \mathrm{O}$ contact to alumina resulting in a sensitive response of capacitance.

The vertical magnetic field was expected to give electro-migration of cation and anion during the anodizing process. During pore formation, the barrier film of alumina is first formed on the surface of $\mathrm{Al}$ substrate. The barrier layer is nonconductive; thus a much higher electric field is needed to break the barrier in certain areas. In the absence of a magnetic field, the non-uniform electric field leads to random sites for pore formation because electric field distribution prefers sites with lower resistivity. The magnetic convection can either promote or suppress the reaction rate, depending on the direction of the magnetic field. ${ }^{(22)}$ It should be noted that the anodizing process is related to the migration of anions $\left(\mathrm{O}^{2-}\right.$ and $\left.\mathrm{OH}^{-}\right)$from the electrolyte and cations $\left(\mathrm{Al}^{3+}\right)$ through the alumina. If a vertical magnetic field is applied, during pore formation induced by anodization, micro MHD convection is widely distributed at pore initiation sites, leading to higher pore density. This MHD effect however, suppresses the anodic reaction, as some of the $\mathrm{O}^{2-}, \mathrm{OH}^{-}$, and $\mathrm{Al}^{3+}$ ions may be affected by the convection, resulting in a low electric field along the alumina for pore formation. As the magnetic field increases, the effect become stronger, which is why the pore diameter decreases. As the pore begins to grow, the pore size is maintained without further increase of pore size.

Normally, the MHD effect will be generated when the magnetic field is perpendicular to the current direction. However, it appears that in the region between the alumina and the electrolyte interface, the MHD and micro-MHD are generated where the current direction and magnetic field is not perfectly parallel. This kind of convection has been reported by Aogaki et al. in copper electrodeposition. ${ }^{(19,20)}$ Bund et al. also reported this magnetic convection because of the gradient of magnetic susceptibility in the solution, especially between the bulk and Nernst layer in copper electrodeposition. ${ }^{(17)}$ In this experiment, during the porous growth, the micro MHD affects the ionic 
migration, $\mathrm{O}^{2-}, \mathrm{OH}^{-}, \mathrm{Al}^{3+}$, during oxide dissolution for pore growth in a circular manner along the pore channel. The convection arises at the vicinity between the alumina and electrolyte interface. This convection is generated because of the interaction of an electric field from aluminum to alumina which flows to the pore initiation sites so that the current direction is not perfectly parallel to the magnetic field. As the pore formed and started to grow downward, the magnetic convection affected the ion transfer of cations $\left(\mathrm{OH}^{-}\right.$and $\left.\mathrm{O}^{2-}\right)$ from the electrolyte to alumina, maintained in circular manner. Hence, the release of $\mathrm{Al}^{3+}$ sites is also maintained in a circular manner in the alumina-electrolyte interface. Therefore, the AAO pores maintain growth with a circular shape; thus a vertical aligned cylindrical pore structure can be produced even in a magnetic field of $1 \mathrm{~T}$. However as the magnitude of the magnetic field increases, this circular convection induces only the migration of cations and anions but does not affect the size of the pore channel during downward growth of the pores. The increases of porous thickness of AAO formed with imposed magnetic field show that micro MHD could increase ion transfer during anodic dissolution of $\mathrm{Al}^{3+}$ from alumina to electrolyte during growth of the pores. It seems that the increasing magnitude of the magnetic field does not significantly increase the ion transfer, so the thickness is relatively constant with increasing magnetic field.

The XRD patterns of the AAO formed in various magnitudes of magnetic field is presented in Fig. 9. The aluminum diffraction patterns are inserted, as shown in Fig. 9, which was the International Centre for Diffraction Data (ICDD): PDF\#00-001-1176, extracted from PDXL version 2.4.2.0, Rigaku. There were diffraction peaks of aluminum sheet and no diffraction peaks of alumina in the XRD patterns of AAO film formed without imposition of a magnetic field in Fig. 9(a), because AAO film is very thin, as seen in Fig. 8. All the XRD patterns of AAO film formed with the imposition of a magnetic field indicated an amorphous phase, as shown in Figs. 9(b)-9(e), because the X-ray did not reach the bottom aluminum sheet below the thick AAO film, as seen in Fig. 8. The amorphous phase of AAO resulting from anodizing was also in good agreement with previous reports. ${ }^{(6)}$

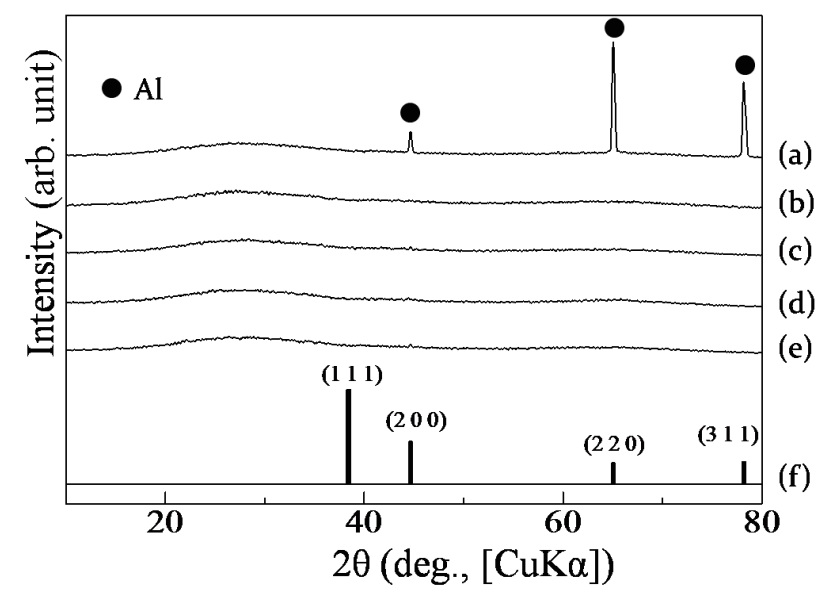

Fig. 9. XRD patterns of the AAO formed by anodizing (a) without magnetic field and with the magnetic field of (b) 1 , (c) 2, (d) 3, and (e) $5 \mathrm{~T}$; (f) the ICDD data of aluminum patterns. 


\section{Conclusion}

The effect of magnetic field imposition was examined on aluminum anodizing behavior in order to understand the metal oxidation and pore formation phenomena in a metal anodizing process. The results obtained are summarized as follows.

1. An AAO layer with dense nanopores on the surface was prepared by magnetic field imposition during anodizing of aluminum.

2. An AAO layer with cylindrical morphology in the cross-section was formed by a magnetic field ranging from 1 to $5 \mathrm{~T}$ which was perpendicularly imposed on the aluminum substrate during the anodizing of aluminum in a single step.

3. The role of the MHD effect is guiding the alumina growth vertically by affecting the migration of ions and increasing ion transfer during porous growth.

4. The pore diameter of AAO was suppressed by increasing the magnitude of the magnetic field.

5. The AAO film formed with a magnetic field has a deep and straight pore structure which contributes to the increase in the adsorption amount of gases due to a larger specific area of AAO.

\section{Acknowledgements}

The authors are indebted to the Monbukagakusho Scholarship from the Ministry of Education, Culture, Sports, Science and Technology (MEXT) of Japan for providing financial support during the study at Kumamoto University, Japan. This work was partially supported by a Grant-in-Aid for Scientific Research, KAKENHI (25246013).

\section{References}

1 G. E. Thompson: Thin Solid Films 297 (1997) 192.

2 T. Wu, T. Meen, L. Ji, S. Chao, C. Huang, J. Tsai, T. Wu, and C. Huang: Sens. Mater. 28 (2016) 539.

D. Eun, J. Jeong, J. Shin, and J. Lee: Sens. Mater. 20 (2008) 397.

4 T. Hashishin, Y. Tono, and J. Tamaki: Jpn. J. Appl. Phys. 45 (2006) 333.

5 P. Koedrith, T. Thasipu, K. Tuitemwong, R. Boonprasert, and P. Tuitemwong: Sens. Mater. 27 (2015) 1113.

6 G. D. Sulka: Nanostructured Materials in Electrochemistry, ed. A. Eftekhari (Wiley-VCH, Germany, 2008) p. 1.

7 A. Jani, D. Losic, and N. Voelcker: Prog. Mater. Sci. 58 (2013) 636.

8 Z. Chen and C. Liu: Sens. Lett. 3 (2005) 274.

9 T. Islam, K. K. Mistry, K. Sengupta, and H. Saha: Sens. Mater. 7 (2004) 345.

10 Q. Fu, C. Cao, and H. Zhu: Thin Solid Films 348 (1999) 99.

11 L. P. H. Jeurgens, W. G. Sloof, F. D. Tichelaar, and E. J. Mittemeijer: Thin Solid Films 418 (2002) 89.

12 O. Jessensky, F. Muller, and U. Gosele: J. Electrochem. Soc. 145 (1998) 3735.

13 W. J. Stępniowski and Z. Bojar: Surf. Coat. Tech. 206 (2011) 265.

14 H. Masuda and K. Fukuda: Science 268 (1995) 1466.

15 G. D. Sulka and W. J. Stępniowski: Electrochim. Acta 54 (2009) 3683.

16 S. Asai: Proc. 4th IC Electromagnetic Processing of Materials 2003, eds. S. Asai, Y. Fautrelle, P. Gillon, and F. Durand (EPM Madylam, Lyon, 2003) pp. 1-8.

17 A. Bund, S. Koehler, H. H. Kuehnlein, and W. Plieth: Electrochim. Acta 49 (2003) 147.

18 T. Kozuka, S. Sakurai, and M. Kawahara: Proc. 6th IC Electromagnetic Processing of Materials 2009, eds. G. Gerbeth and Y. Fautrelle (Forschungszentrum Dresden-Rossendorf, Dresden, 2009) pp. 431-434.

19 R. Aogaki: Magnetohydrodynamics 39 (2003) 453.

20 R. Morimoto, S Yazawa, R. Aogaki, A. Sugiyama, and M. Saitou: J. Surf. Finish. Soc. Jpn. 59 (2008) 408 (in Japanese).

21 M. Takezawa, T. Imagawa, J. Yamasaki, and M. Yagi: Sens. Lett. 5 (2007) 293.

22 T. Kozuka, T. Sakai, R. Miyamura, and M. Kawahara: ISIJ International 43 (2003) 884. 